\title{
TRAPPING ROSSBY WAVES
}

\author{
CHRISTOPHE CHEVERRY, ISABELLE GALLAGHER, THIERRY PAUL, \\ AND LAURE SAINT-RAYMOND
}

\begin{abstract}
Waves associated to large scale oceanic motions are gravity waves (Poincaré waves which disperse fast) and quasigeostrophic waves (Rossby waves). In this Note, we show by semiclassical arguments, that Rossby waves can be trapped and we characterize the corresponding initial conditions.
\end{abstract}

\section{INTRODUCTION AND RESULTS}

Large scale oceanic motions can be described - at first sight - by the linearized Saint-Venant equations for thin layers, with Coriolis force, i.e. by the following two-dimensional system [6]:

(1)

$\partial_{t} U+\left(\begin{array}{ccc}0 & \partial_{x_{1}} & \partial_{x_{2}} \\ \partial_{x_{1}} & 0 & -B\left(x_{2}\right) \\ \partial_{x_{2}} & B\left(x_{2}\right) & 0\end{array}\right) U=0$, where $U=U\left(t, x_{1}, x_{2}\right)=\left(\begin{array}{c}\rho \\ u_{1} \\ u_{2}\end{array}\right)$

where $B$ is the local vertical component of the Earth rotation vector (depending only on the latitude $x_{2}$ ), $\rho$ denotes the fluctuation of water height and $u$ the horizontal velocity field. For the sake of simplicity, we assume that $\left(x_{1}, x_{2}\right) \in \mathbb{R} \times \mathbb{T}$, meaning that we neglect the influence of the lateral boundaries.

Waves associated to that linear system are usually classified in two families : First, Poincaré waves, which are fast dispersive gravity waves; Second, Rossby waves, due to the inhomogeneities of $B$, which propagate much slower [2], [3].

In this Note, we are interested in the propagation of Rossby waves. We show, in agreement with physical observations [7], that these waves can be trapped in some regions, called ventilation zones, which are not influenced by external dynamics and sources such as continental recirculation for instance.

More precisely, in the limit of large values of $B=b / \epsilon$, we construct a co-dimension 1 submanifold $\Lambda$ of $T^{*}(\mathbb{R} \times \mathbb{T})$. This set contains the $\epsilon$ microlocalization region inside which, essentially, an initial condition of (1) remains trapped. Let us first recall that the $\epsilon$-frequency set of a function $u$ [4] is the (closed) subset of $T^{*}(\mathbb{R} \times \mathbb{T})$, complement of the set of points $(\underline{x}, \underline{\xi})$ such that there exists a $C^{\infty}$ function $\chi$, with $\chi(\underline{x}, \underline{\xi})=1$, such that

$$
\left\|\int \chi\left(\frac{x+y}{2}, \xi\right) e^{i \frac{\xi(x-y)}{\epsilon}} u(y) d y d \xi\right\|_{L^{2}}=O\left(\epsilon^{\infty}\right) .
$$


Theorem 1.1. Let us consider the system

(2) $\partial_{t} U+\left(\begin{array}{ccc}0 & \partial_{x_{1}} & \partial_{x_{2}} \\ \partial_{x_{1}} & 0 & -\frac{b\left(x_{2}\right)}{\epsilon} \\ \partial_{x_{2}} & \frac{b\left(x_{2}\right)}{\epsilon} & 0\end{array}\right) U=0$ with an initial condition

$$
\left.U\right|_{t=0}=\left(\begin{array}{c}
\rho^{0} \\
u_{1}^{0} \\
u_{2}^{0}
\end{array}\right)
$$

with $L^{2}\left(\mathbb{R} \times \mathbb{T}, d x_{1} d x_{2}\right)$ conditions (periodic in $\left.x_{2}\right)$. Let $\Lambda=\left\{F\left(\xi_{1}, x_{2}, \xi_{2}\right)=\right.$ $0\} \subset T^{*} \mathbb{R} \times \mathbb{T}$, where $F$ is defined in Lemma 3.1.

We suppose that the $\epsilon$-frequency set of $\left.U\right|_{t=0}$ is contained in a compact set $\mathcal{C}$ satisfying:

$$
\mathcal{C} \cap\left\{\xi_{1}=0\right\}=\mathcal{C} \cap\left\{\xi_{2}^{2}+b\left(x_{2}\right)^{2}=0\right\}=\emptyset .
$$

Let us fix a compact set $\Omega$ of $\mathbb{R} \times \mathbb{T}$. Then there exists $(\epsilon$-)pseudo-differential operators $P_{\rho}^{0}, P_{1}^{0}, P_{2}^{0}$ of principal symbols $p_{\rho}^{0}=i b\left(x_{2}\right) \xi_{1}\left(\xi_{2}^{2}+\xi_{1}^{2}+b^{2}\left(x_{2}\right)\right)^{-1}$, $p_{1}^{0}=-\xi_{1} \xi_{2}\left(\xi_{2}^{2}+\xi_{1}^{2}+b^{2}\left(x_{2}\right)\right)^{-1}, p_{2}^{0}=\xi_{1}^{2}\left(\xi_{2}^{2}+\xi_{1}^{2}+b^{2}\left(x_{2}\right)\right)^{-1}$, such that:

(1) if the $\epsilon$-frequency set of $P_{\rho}^{0} \rho^{0}+P_{1}^{0} u_{1}^{0}+P_{2}^{0} u_{2}^{0}$ does not intersect $\Lambda \cap$ $T^{*} \Omega$, then $\exists T>0$ such that:

$$
\left\|U\left(\frac{t}{\epsilon}\right)\right\|_{L^{2}(\Omega)}=O\left(\epsilon^{\infty}\right) \text { for } t \geq T
$$

(2) if the $\epsilon$-frequency set of $P_{\rho} \rho^{0}+P_{1} u_{1}^{0}+P_{2} u_{2}^{0}$ does intersect $\Lambda \cap T^{*} \Omega$, then, $\forall t \geq 0,\left\|U\left(\frac{t}{\epsilon}\right)\right\|_{L^{2}(\Omega)} \neq O\left(\epsilon^{\infty}\right)$ (in other words the frequency set of $U\left(\frac{t}{\epsilon}\right)$ intersects $\left.T^{*} \Omega\right)$.

In the case of a WKB initial condition the conclusion is more precise. Consider

$$
\left.U\right|_{t=0}=\left(\begin{array}{c}
R^{0}(x) \\
U_{1}^{0}(x) \\
U_{2}^{0}(x)
\end{array}\right) e^{i \frac{S(x)}{\epsilon}}
$$

Suppose than the (Lagrangian) manifold $\left\{(x, \nabla S(x)),\left(p_{\rho}^{0} R^{0}+p_{1}^{0} U_{1}^{0}+p_{2}^{0} U_{2}^{0}\right)(x, \nabla S(x)) \neq 0\right\}$ intersects $\Lambda \cap T^{*} \Omega$, then:

$$
\left\|U\left(\frac{t}{\epsilon}\right)\right\|_{L^{2}(\Omega)} \sim C(t)+O(\epsilon), C(t)>0
$$

\section{REduction to a scalar Situation}

Performing first a Fourier analysis in $x_{1}$ (as the system does not contain explicitly this variable) and looking secondly at modes of the system (2), we can prove the following Proposition, heart of our results:

Proposition 2.1. There exist three pseudo-differential operators $T_{ \pm}, T_{0}$, of leading symbols 
$\tau_{ \pm}= \pm \sqrt{\xi_{1}^{2}+\xi_{2}^{2}+b^{2}\left(x_{2}\right)}, \tau_{0}=\epsilon \frac{b^{\prime}\left(x_{2}\right) \xi_{1}}{\xi_{1}^{2}+\xi_{2}^{2}+b^{2}\left(x_{2}\right)}$, roots of (\&), such that, if $u_{2, j}^{0}$ is microlocalized outside $\xi_{1}^{2}-\tau_{j}^{2}=0$, we have, for $j \in\{-, 0,+\}$ :

$$
\epsilon \partial_{t} u_{2, j}=i T_{j} u_{2, j} \Longrightarrow U_{j}:=\left(\begin{array}{c}
\left(i \epsilon \partial_{x_{2}} T_{j}+\epsilon \partial_{x_{1}} b\left(x_{2}\right)\right)\left(\epsilon^{2} \partial_{x_{1}}^{2}+T_{j}^{2}\right)^{-1} \\
-\left(\epsilon^{2} \partial_{x_{1}} \partial_{x_{2}}+i b\left(x_{2}\right) T_{j}\right)\left(\epsilon^{2} \partial_{x_{1}}^{2}+T_{j}^{2}\right)^{-1} \\
\mathbb{I} d
\end{array}\right) u_{2, j}
$$

The proof is a consequence of the result contained in the Appendix.

The following result shows that any initial condition of (2) can be decomposed on the three modes of the last section.

Proposition 2.2. $\forall \rho, u_{1}, u_{2}, \exists u_{2, j}, j \in\{+, 0,-\}$ such that:

(6)

$$
\begin{gathered}
\left(\begin{array}{c}
\rho \\
u_{1} \\
u_{2}
\end{array}\right)=\sum_{j}\left(\begin{array}{c}
\left(i \epsilon \partial_{x_{2}} T_{j}+\epsilon \partial_{x_{1}} b\left(x_{2}\right)\right)\left(\epsilon^{2} \partial_{x_{1}}^{2}+T_{j}^{2}\right)^{-1} \\
-\left(\epsilon^{2} \partial_{x_{1}} \partial_{x_{2}}+i b\left(x_{2}\right) T_{j}\right)\left(\epsilon^{2} \partial_{x_{1}}^{2}+T_{j}^{2}\right)^{-1} \\
\mathbb{I} d
\end{array}\right) u_{2, j}+O\left(\epsilon^{\infty}\right) \\
=: \sum_{j} \mathbb{Q}^{j} u_{2, j}+O\left(\epsilon^{\infty}\right) .
\end{gathered}
$$

To prove the Proposition one has just to invert the matrix: $\left(\mathbb{Q}^{-} \mathbb{Q}^{0} \mathbb{Q}^{+}\right)$. Semiclassically it is enough to show that the matrix of the leading order symbol is invertible

$$
\left(\begin{array}{ccc}
\frac{\xi_{2} \sqrt{\xi_{1}^{2}+\xi_{2}^{2}+b\left(x_{2}\right)^{2}}+i \xi_{1} b\left(x_{2}\right)}{\xi_{2}^{2}+b\left(x_{2}\right)^{2}} & -\frac{i b\left(x_{2}\right)}{\xi_{1}} & \frac{-\xi_{2} \sqrt{\xi_{1}^{2}+\xi_{2}^{2}+b\left(x_{2}\right)^{2}}+i \xi_{1} b\left(x_{2}\right)}{\xi_{2}^{2}+b\left(x_{2}\right)^{2}} \\
\frac{\xi_{1} \xi_{2}+i b\left(x_{2}\right) \sqrt{\xi_{1}^{2}+\xi_{2}^{2}+b\left(x_{2}\right)^{2}}}{\xi_{2}^{2}+b\left(x_{2}\right)^{2}} & -\frac{\xi_{2}}{\xi_{1}} & \frac{\xi_{1} \xi_{2}-i b\left(x_{2}\right) \sqrt{\xi_{1}^{2}+\xi_{2}^{2}+b\left(x_{2}\right)^{2}}}{\xi_{2}^{2}+b\left(x_{2}\right)^{2}} \\
1 & 1 & 1
\end{array}\right) .
$$

A simple computation shows that the jacobian $J=\frac{2\left(\xi_{1}^{2}+\xi_{2}^{2}+b^{2}\left(x_{2}\right)\right)^{3 / 2}}{\left(\xi_{2}^{2}+b^{2}\left(x_{2}\right)\right)\left|\xi_{1}\right|} \geq 2$. In particular the inversion of the matrix $\left(\mathbb{Q}^{-} \mathbb{Q}^{0} \mathbb{Q}^{+}\right)$can be done symbolically at any order and gives the leading order symbols of the operators $\mathbb{P}^{j}:=$ $\left(P_{\rho}^{j}, P_{1}^{j}, P_{2}^{j}\right)$ such that $u_{2, j}=P_{\rho}^{j} \rho+P_{1}^{j} u_{1}+P_{2}^{j} u_{2}, j \in\{-, 0,+\}$. One gets : $p_{\rho}^{0}=i b\left(x_{2}\right) \xi_{1}\left(\xi_{2}^{2}+\xi_{1}^{2}+b^{2}\left(x_{2}\right)\right)^{-1}, p_{1}^{0}=-\xi_{1} \xi_{2}\left(\xi_{2}^{2}+\xi_{1}^{2}+b^{2}\left(x_{2}\right)\right)^{-1}, p_{2}^{0}=$ $\xi_{1}^{2}\left(\xi_{2}^{2}+\xi_{1}^{2}+b^{2}\left(x_{2}\right)\right)^{-1}$.

\section{Propagation under the Rossby Hamiltonian $\tau_{0}$}

Thanks to Proposition 2.1 it is enough, as far as the Rossby mode is concerned, to look at propagation with respect to the Hamiltonian $\tau_{0} / \epsilon$. This Hamiltonian being independent of $x_{1}, \xi_{1}$ will be conserved. The flow is periodic in the variables $x_{2}, \xi_{2}$ (one degree of freedom). Therefore, since $\dot{x}_{1}=$ $\frac{b^{\prime}\left(x_{2}\right)\left(\xi_{2}^{2}-\xi_{1}^{2}+b^{2}\left(x_{2}\right)\right)}{\left(\xi_{2}^{2}+\xi_{1}^{2}+b^{2}\left(x_{2}\right)\right)^{2}}$ is periodic (the case of infinite and zero period is treated in [1]), $x_{1}(t)$ will contain a part, linear in time except if

$$
F\left(\xi_{1}, x_{2}(0), \xi_{2}(0)\right):=\int_{0}^{p e r i o d} \frac{b^{\prime}\left(x_{2}(t)\right)\left(\xi_{2}(t)^{2}-\xi_{1}^{2}+b^{2}\left(x_{2}(t)\right)\right)}{\left(\xi_{2}(t)^{2}+\xi_{1}^{2}+b^{2}\left(x_{2}(t)\right)\right)^{2}} d t=0 .
$$


Lemma 3.1. As $b^{\prime}\left(x_{2}\right) \neq 0, b^{\prime}\left(x_{2}\right) F\left(\xi_{1}, x_{2}, \xi_{2}\right)>0$ as $\xi_{1} \rightarrow \pm \infty,<0$ as $\xi_{1} \rightarrow 0$ and is invariant under the flow of $\frac{\tau_{0}}{\epsilon}$.

Define $E\left(\xi_{1}, x_{2}, \xi_{2}\right)=\frac{b^{\prime}\left(x_{2}\right) \xi_{1}}{\xi_{2}^{2}+\xi_{1}^{2}+b^{2}\left(x_{2}\right)}$.

Then $\left|F\left(\xi_{1}, x_{2}, \xi_{2}\right)\right|=\left|\int_{x_{-}}^{x_{+}} \frac{\frac{b^{\prime}(x)}{E\left(\xi_{1}, x_{2}, \xi_{2}\right)}-2 \xi_{1}}{\sqrt{\frac{b^{\prime}(x) \xi_{1}}{E\left(\xi_{1}, x_{2}, \xi_{2}\right)}-\xi_{1}^{2}-b^{2}(x)}} d x\right|$, where $] x_{-}, x_{+}[$ is the largest interval of $\mathbb{T}$ containing $x_{2}$ in which $\frac{b^{\prime}(x) \xi_{1}}{E\left(\xi_{1}, x_{2}, \xi_{2}\right)}-\xi_{1}^{2}-b^{2}(x)>0$.

We define $\Lambda:=\left\{\left(x_{1}, x_{2} ; \xi_{1}, \xi_{2}\right) / F\left(\xi_{1}, x_{2}, \xi_{2}\right)=0\right\}$.

Thanks to Lemma $3.1 \Lambda \neq \emptyset$ and $\operatorname{dim} \Lambda=3$.

Corollary 3.2. Suppose $b^{\prime}\left(x_{2}\right) \neq 0$. Then $\left|F\left(\xi_{1}, x_{2}, \xi_{2}\right)\right| \geq \frac{C\left(x_{2}, \xi_{2}\right)}{\xi_{1}}$ as $\xi_{1} \rightarrow$ 0 , with $C\left(x_{2}, \xi_{2}\right)>0$. This implies that the trapping phenomenon will take place only with initial conditions oscillating enough in $x_{1}$.

Remark 1. : Since the Hamiltonian $E$ does not depend on $x_{1}$, we can express it, for each value of $\xi_{1}$, on the action variable $A$ : $E\left(\xi_{1}, x_{2}, \xi_{2}\right)=$ $H\left(A, \xi_{1}\right)$. This allows to define the function $A\left(\xi_{1}, x_{2}, \xi_{2}\right)$ by $E\left(\xi_{1}, x_{2}, \xi_{2}\right)=$ $H\left(A\left(\xi_{1}, x_{2}, \xi_{2}\right), \xi_{1}\right)$. One can easily show that:

$$
F\left(\xi_{1}, x_{2}, \xi_{2}\right)=\left.\frac{\partial_{\xi_{1}} H\left(A, \xi_{1}\right)}{\partial_{A} H\left(A, \xi_{1}\right)}\right|_{A=A\left(\xi_{1}, x_{2}, \xi_{2}\right)},
$$

and the following variational characterization of $\Lambda$ : Let us fix the energy to $E$ and let $\Gamma\left(\xi_{1}, E\right)$ be the energy shell $\left\{E\left(\xi_{1}, x_{2}, \xi_{2}\right)=E\right\}$. Then

$$
\Lambda=\bigcup_{E, i}\left\{\left(\Gamma\left(\xi_{1}^{i}, E\right), \xi_{1}^{i}\right\}\right.
$$

where $\xi_{1}^{i}$ is such that the area inside $\Gamma\left(\xi_{1}^{i}, E\right)$ is extremal.

\section{Dispersion of Poincaré waves and proof of the Theorem}

The proof of the Theorem involves, for the Rossby modes $(j=0)$, the standard result of propagation of the frequency set. If the initial frequency set is such that part of the Rossby mode is trapped, in particular if it intersects $\left\{b^{\prime}\left(x_{2}\right)=0\right\}$, this concludes the proof.

If not we have to prove some dispersion for the Poincaré modes $(j= \pm)$ for times of the order $\frac{1}{\epsilon}$, for which the theorem of propagation of the frequency set is not enough. But, the system being integrable, we can perform an expansion on (Bohr-Sommerfeld) eigenvalues of the Hamiltonians $T_{ \pm}$and a decomposition of $\left.U\right|_{t=0}$ on a compact set of coherent states (thanks to the condition on the microlocalization of the initial datum) [5].

Since $\tau_{ \pm}= \pm \sqrt{\xi_{2}^{2}+b^{2}\left(x_{2}\right)+\xi_{1}^{2}}+O(\epsilon)$ and we are microlocalized far away from $\xi_{2}^{2}+b^{2}\left(x_{2}\right)+\xi_{1}^{2}=0$, we can find pseudo-differential operators $H_{2 \pm}$ of principal symbols $\xi_{2}^{2}+b^{2}\left(x_{2}\right)$ such that $T_{ \pm}= \pm \sqrt{H_{2 \pm}+\xi_{1}^{2}}$. The 
Bohr-Sommerfeld quantization condition (with subsymbol) gives that the eigenvalues of $H_{2 \pm}$ are of the form:

$$
\lambda_{ \pm}^{k}=\lambda_{ \pm}\left(\left(k+\frac{1}{2}\right) \epsilon\right)+\epsilon \mu_{ \pm}^{k}\left(\xi_{1}\right)+O\left(\epsilon^{2}\right)
$$

where $\lambda_{ \pm}$is the energy $\xi_{2}^{2}+b^{2}\left(x_{2}\right)$ defined on action variable, and $\epsilon \mu_{ \pm}^{k}\left(\xi_{1}\right) \epsilon$ $C^{\infty}$ is the correction due to the subsymbol. Propagating at time $t=s / \epsilon$ a function, product of a coherent state at $(q, p)$ (in $x_{1}$ ) and an eigenfunction of $T_{ \pm}$(in $x_{2}$ ), gives rise to expressions of the type:

$$
\int \exp i \frac{\epsilon\left(x_{1}-q\right) \xi_{1} \pm\left(\lambda_{ \pm}^{k}+\xi_{1}^{2}\right)^{\frac{1}{2}} s+i \epsilon\left(\xi_{1}-p\right)^{2}}{\epsilon} d \xi_{1} .
$$

The stationary phase lemma then gives that this integral is $O\left(\epsilon^{\infty}\right)$ except if there exists a stationary point, given by the conditions:

$$
\xi_{1}=p \text { and } \epsilon\left(x_{1}-q\right) \pm \frac{\left(2 \xi_{1}+\epsilon \partial_{\xi_{1}} \mu_{ \pm}^{k}\right)}{2 \sqrt{\lambda_{ \pm}^{k}+\xi_{1}^{2}}} s=0 .
$$

The second condition gives: $2 \sqrt{\lambda_{ \pm}^{k}+\xi_{1}^{2}}\left(x_{1}-q\right)=\mp\left(\frac{2 p}{\epsilon}+\partial_{\xi_{1}} \mu_{ \pm}^{k}\right) s$. Therefore, since $p \neq 0$ and the $\lambda^{k}$ 's are bounded by the above condition (4) on $\mathcal{C}$, there is no critical point for $x_{1}$ in a compact set.

\section{Appendix A. A microlocal analysis Lemma}

In this Appendix we give the crucial lemma for the proof of Proposition 2.1. It tells us that the principal symbols of $T_{ \pm}, T_{0}$ can be computed by solving the symbolic equation associated to

$$
\left(\epsilon^{2} \partial_{x_{2}}^{2}-b^{2}\left(x_{2}\right)-i \epsilon^{2} \frac{b^{\prime}\left(x_{2}\right) \partial_{x_{1}}}{\tau}+\tau^{2}+\epsilon^{2} \partial_{x_{1}}^{2}\right) u_{2}=0
$$

obtained by (exact) algebraic computations from (11), that is

$$
\tau^{2}-\xi_{1}^{2}-\xi_{2}^{2}-b^{2}+\epsilon \frac{b^{\prime} \xi_{1}}{\tau}=0
$$

Lemma A.1. Let $h=h(x, \xi, \sigma)$ be a smooth function such that $\left.\partial_{\sigma} h\right|_{h=0} \neq 0$, and let $\tau=\tau(x, \xi)$ be any continuous root of $h(x, \xi, \tau(x, \xi))=0$.

Then there exists a pseudo-differential operator $T=T\left(x,-i \epsilon \partial_{x}\right)$ with principal symbol $\tau(x, \xi)$ such that:

$$
T \psi=\lambda \psi \Longrightarrow H(\lambda) \psi=O\left(\epsilon^{\infty}\right)
$$

where $H(\lambda)$ is a pseudo-differential operator of full symbol $h(x, \xi, \lambda)$.

Sketch of the proof: the proof uses essentially pseudo-differential functional calculus [四].

For any $(x, \xi)$ the principal symbol of $h(x, \xi, T)$ at $\left(y, \xi^{\prime}\right)$ is $h\left(x, \xi, \tau\left(y, \xi^{\prime}\right)\right)$. We can write at first order: $H \psi:=\int e^{i \frac{\xi(x-y)}{\epsilon}} h\left(x, \xi, T\left(y,-i \epsilon \partial_{y}\right)\right) \psi(y) \frac{d \xi d y}{\epsilon}=$ 
$\int e^{i \frac{\xi(x-y)}{\epsilon}} e^{i \frac{\xi^{\prime}\left(y-y^{\prime}\right)}{\epsilon}} h\left(x, \xi, \tau\left(y^{\prime}, \xi^{\prime}\right)\right) \psi\left(y^{\prime}\right) \frac{d \xi d \xi^{\prime} d y}{\epsilon}$. The integral over $y$ gives $\delta(\xi-$ $\left.\xi^{\prime}\right)$. Therefore: $H \psi(x)=\int e^{i \frac{\xi(x-y)}{\epsilon}} h(x, \xi, \tau(y, \xi)) \psi(y) \frac{d \xi d y}{\epsilon}$. So the principal symbol of $H$ is $h(x, \xi, \tau(x, \xi))$ which, by assumption, is 0 .

For the $\epsilon^{\infty}$ result, it is enough to repeat the same argument taking into account lower order terms $\epsilon^{k} \tau_{k}$ for and adding to any symbol a term of the form $\sum_{k \geq 1} \epsilon^{k} P_{k} \tau$ where the $P_{k}$ s are differential operators. We end up with an equation for $\tau_{\epsilon} \sim \tau+\sum \epsilon^{k} \tau_{k}$ of the form:

$h\left(x, \xi, \tau_{\epsilon}\right)+\sum_{k \geq 1} \epsilon^{k} Q_{k}\left(\tau, \ldots, \partial_{x}^{l} \partial_{\xi}^{m} \tau_{\epsilon}\right)=0$, that can be solved recursively under the condition $\left.\partial_{\tau} h(x, \xi, \tau)\right|_{h(x, \xi, \tau)=0} \neq 0$.

\section{ACKNOWLEDGEMENTS}

We would like to thank Agis Athanassoulis and Bach-Lien Hua for helpful discussions.

\section{References}

[1] C. Cheverry, I. Gallagher, T. Paul and L. Saint-Raymond, in preparation.

[2] A. Dutrifoy, A. J. Majda and S. Schochet, A Simple Justification of the Singular Limit for Equatorial Shallow-Water Dynamics, in Communications on Pure and Applied Math. LXI (2008) 0002-0012.

[3] I. Gallagher and L. Saint-Raymond, Mathematical study of the betaplane model: equatorial waves and convergence results. Mém. Soc. Math. Fr. (N.S.). 107 (2006), $\mathrm{v}+116 \mathrm{pp}$.

[4] A. Martinez, An introduction to semiclassical and microlocal analysis, Springer (2002)

[5] T. Paul, Échelles de temps pour l'évolution quantique à petite constante de Planck, Séminaire X-EDP 2007-2008, École polytechnique.

[6] J. Pedlosky, Geophysical fluid dynamics, Springer (1979).

[7] J. Pedlosky, Ocean Circulation Theory, Springer (1996).

E-mail address: christophe.cheverry@univ-rennes1.fr

E-mail address: gallagher@math.jussieu.fr

E-mail address: thierry.paul@ens.fr

E-mail address: Laure. Saint-Raymond@ens.fr

(Cheverry) Institut Mathématique de Rennes, Campus de Beaulieu, 263 AVenue du Général LeClerc CS 7420535042 Rennes Cedex

(Gallagher) Institut de Mathématiques de Jussieu et Université Paris VII, Case 7012, 2 place Jussieu 75251 Paris Cedex 05

(Paul) CNRS and DMA École Normale Supérieure, 45 Rue d'Ulm, 75230 Paris Cedex 05

(Saint-Raymond) Université Paris VI and DMA École Normale Supérieure, 45 Rue D'Ulm, 75230 Paris Cedex 05 